\title{
herbivorous tortoises
}

8 Diana Montes-Grajales ${ }^{1,2}$, Berenice Jiménez ${ }^{1}$, Marco A. Rogel ${ }^{1}$, Alejandro Alagón ${ }^{3}$,

9 Nuria Esturau-Escofet ${ }^{4}$, Baldomero Esquivel ${ }^{4}$, Julio Martínez-Romero ${ }^{1}$ and Esperanza

10 Martínez-Romero ${ }^{1 *}$.

13 1. Centro de Ciencias Genómicas, Universidad Nacional Autónoma de México,

14 Cuernavaca, Mexico.

152 . Environmental and Computational Chemistry Group, School of Pharmaceutical

16 Sciences, University of Cartagena, Cartagena, Colombia.

17 3. Biotechnology Institute, Universidad Nacional Autónoma de México, Cuernavaca,

18 Mexico.

19 4. Instituto de Química, Universidad Nacional Autónoma de México, Mexico City,

20 Mexico.

21

$23 *$ Corresponding autor

24 E-mail: emartine@ccg.unam.mx (EM-R) 


\section{Abstract}

Animals feeding on plants (herbivorous) may have nutritional deficiencies and

27 use bacterial nitrogen fixation in guts to compensate unbalanced diets with high carbon

28 and low nitrogen. Using the acetylene reduction assay we searched for nitrogen fixation

29 in the feces from several herbivorous animals in captivity. We detected acetylene

30 reduction in feces from two African spurred tortoises, Centrochelys sulcata and in feces

31 from six Gopherus berlandieri tortoises and isolated nitrogen-fixing klebsiellas from

32 them. Additionally, we performed a gut metagenomic study with Illumina sequencing

33 from a healthy Mexican G. berlandieri tortoise, and the nif genes identified in the feces

34 microbiome matched those from Klebsiella variicola. Fecal bacterial composition from

35 tortoises was similar to that reported from other reptilian guts. 


\section{Introduction}

Nitrogen fixation is a unique biochemical process carried out by nitrogenases encoded by nif genes, which are found in only a few prokaryotes (1). Nitrogen fixation is energetically expensive and nitrogen-fixing bacteria are in many cases minor components of the microbial community that nevertheless provide a valuable ecological service (2). Nitrogen-fixing bacteria are called diazotrophs (3).

When associated with nitrogen-fixing bacteria, insects or plants may inhabit in nitrogen poor conditions. Termites contain nitrogen-fixing bacteria in their guts that allow them to grow in wood, similarly wood eating beetles contain nitrogen-fixing bacteria (4). Nitrogen fixation by Klebsiella variicola occurs in the fungal garden of ants (5). A novel betaproteobacterium capable of fixing nitrogen is a recently described symbiont that is transmitted by eggs of carmine cochineals which feed on cacti as the Gopherus berlandieri tortoise does (6). Cacti have high sugar content and are nitrogen poor, conditions that would be favorable for nitrogen fixation in animals that use cacti for food, such as the carmine cochineal or the G. berlandieri tortoise.

G. berlandieri, is distributed from North Mexico (states of Coahuila, Nuevo Leon and Tamaulipas) to South-central Texas in United States (7). This species generally maintains restricted mobility by living for prolonged times in the same burrow (8) and its diet is mainly composed of grasses, weeds and cacti. On the other hand, the African spurred tortoise, Centrochelys sulcata, which is also vegetarian, lives in West Africa (9).

Metagenomic analyses have contributed to gain a better insight on taxonomic composition and functions of the gut microbiota of various species, including nonmodel organisms, such as sea turtles (10). Gut microbiota may confer adaptability to herbivorous tortoises such as $G$. berlandieri and $C$. sulcata naturally exposed to low 
nitrogen-content diets. The aim of this research was to identify nitrogen-fixing species in the gut microbiota of $G$. berlandieri and $C$. sulcata. In addition we present one $G$. berlandieri fecal metagenome analysis and nuclear magnetic resonance (NMR) spectroscopy-based identification of the exometabolites produced by one of the nitrogen-fixing bacterial isolates from the gut microbiota of $G$. berlandieri and by $K$. variicola F2R9 as reference.

\section{Materials and methods}

\section{Samples}

Six samples -each from a different individual- were obtained from $G$. berlandieri turtles kindly supplied by Ezer Yniestra with permission of SEMARNAT. They were fed with a vegetarian diet based mainly on cactus cladodes, grass, clover, dandelion and lettuce and received a dietary supplement based on vegetables once a week. They were dewormed annually and never received antibiotics. Another tortoise species with similar habits and life conditions is the African turtle C. sulcata. A $C$. sulcata stool sample was provided by Zoofari local zoo and used to detect acetylene reduction, additional samples were from Dr. Alejandro Alagón's C. sulcata tortoise, which has been also maintained under a vegetarian diet.

All stool samples were collected using sterile falcon tubes and stored at $-20^{\circ} \mathrm{C}$. The unique sample used for metagenomic DNA extraction and sequencing, consisted of feces from a female adult $G$. berlandieri tortoise from the herpetarium of the National Autonomous University of Mexico Faculty of Sciences, located in Mexico City (Mexico). This followed an herbivorous diet and did not take antibiotics at least the year before the sample was taken. 
Nitrogen-fixing bacteria isolation and acetylene reduction

\section{assays}

89

Samples of feces of six individuals of $G$. berlandieri and one of $C$. sulcata were employed for nitrogen-fixing bacteria isolation and molecular identification, as well as food samples of $G$. berlandieri consisting on a mixture of $85 \%$ of cactus (nopal) and $15 \%$ lettuce. Glass vials of $10 \mathrm{ml}$ were filled with $5 \mathrm{ml}$ of soft agar nitrogen free medium $\left(\mathrm{Na}_{2} \mathrm{HPO}_{4} \cdot 12 \mathrm{H}_{2} \mathrm{O} 0.22 \%\right.$ w/v; $\mathrm{NaH}_{2} \mathrm{PO}_{4} \cdot \mathrm{H}_{2} \mathrm{O} 0.0425 \%$ w/v; $\mathrm{MgSO}_{4} \cdot 7 \mathrm{H}_{2} \mathrm{O} 0.04375 \%$ w/v; $\mathrm{C}_{12} \mathrm{H}_{22} \mathrm{O}_{11} 0.1 \%$; Fahraeus traces $0.1 \% \mathrm{v} / \mathrm{v}$ and agar $0.22 \%$ ). Six replicates of each sample were inoculated and incubated for 2 days into the medium $\left(3\right.$ replicates at $30^{\circ} \mathrm{C}$, and the others at $37^{\circ} \mathrm{C}$ ). After that cotton plugs where changed for hermetic rubber caps, $0.6 \mathrm{ml}$ of acetylene were injected in the flasks and incubated for 3 days. A volume of $0.4 \mathrm{ml}$ of air from each vial was used for ethylene and acetylene detection by gas chromatography (GC). In addition, acetylene reduction activity of the stools samples and isolates were tested using different carbon sources, such as sucrose, glucose and Dgluconic acid sodium salt. presented acetylene reduction and incubated for $24 \mathrm{~h}$ at $30^{\circ} \mathrm{C}$ or $37^{\circ} \mathrm{C}$. Bacteria with different phenotypic features from each sample were isolated by the streak plate method and tested for acetylene reduction activity (ARA) by GC, after $24 \mathrm{~h}$ of growth in glass vials with $5 \mathrm{ml}$ of nitrogen free medium and $24 \mathrm{~h}$ of incubation with $0.6 \mu \mathrm{l}$ of acetylene. A total of 25 bacterial isolates were chosen for DNA extraction and identification of enterobacterial repetitive intergenic consensus (ERIC) PCR patterns. 
111 At least one sample of the purified PCR product of each pattern was used for paired

112 end 16S rDNA gene sequencing (Macrogen). Additionally, a BOX-A1R-based

113 repetitive extragenic palindromic-PCR (BOX-PCR) was performed with few selected 114 isolates.

115

116 Phylogenetic analysis of nitrogen-fixing bacterial isolates

using 16S rRNA gene sequences and antibiotic susceptibility

118 test

119

127

128

131 carbenicillin $100 \mu \mathrm{g} / \mathrm{ml}(\mathrm{Cb} 100)$, nalidixic acid $20 \mu \mathrm{g} / \mathrm{ml}$ (Nal20), tetracycline $10 \mu \mathrm{g} / \mathrm{ml}$

132 (Tc10), chloramphenicol $25 \mu \mathrm{g} / \mathrm{ml}(\mathrm{Cm} 25)$, kanamycin $50 \mu \mathrm{g} / \mathrm{ml}(\mathrm{Km} 50)$, neomycin $133100 \mu \mathrm{g} / \mathrm{ml}$ (Nm100), spectinomycin $100 \mu \mathrm{g} / \mathrm{ml}$ (Sp100), ampicillin $100 \mu \mathrm{g} / \mathrm{ml}$ 134 (Am100), $20 \mu \mathrm{g} / \mathrm{ml}$ gentamicin (Gm20), streptomycin. $100 \mu \mathrm{g} / \mathrm{ml}$ (Sm100). -Controls 135 with no antibiotics were grown. All samples were kept in glass tubes under shaking 
136 (250 rpm), and initial OD600 0.1, during 24h. Subsequently, he relative bacterial

137 growth was measured by comparison with the respective sample.

DNA extraction and metagenome sequencing

141 described by Zhang et al. (16). The ethanol wash was performed on ice followed by

142 centrifugation at $4^{\circ} \mathrm{C}$; lysis was carried out by using liquid nitrogen, and the incubation

143 with $\mathrm{K}$ proteinase was conducted during 4 hours at $45^{\circ} \mathrm{C}$. A 1:1 liquid-liquid extraction

144 was done with phenol by shaking gently for 10 minutes and centrifuging at $3000 \mathrm{~g}$ for

14510 minutes. A second extraction was conducted with phenol:chloroform:isoamyl

146 alcohol 25:24:1 by shaking gently for 10 minutes and centrifuging at $3000 \mathrm{~g}$ for 10

147 minutes. DNA was precipitated using isopropanol $99 \%$ overnight at $-20^{\circ} \mathrm{C}$, followed

148 by centrifugation ( $30 \mathrm{~min}, 8000 \mathrm{~g}$ ) to decant it. The pellet was washed twice with $1 \mathrm{ml}$

149 of $70 \%$ ethanol and dried, then resuspended in $30 \mu \mathrm{l}$ of TE buffer $10 / 1$ and incubated

150 with $10 \mu \mathrm{l}$ of RNasa $(1 \mathrm{mg} / \mathrm{ml})$. To avoid the presence of cetrimonium bromide (CTAB),

151 which has a soapy consistency, a third extraction using phenol:chloroform:isoamyl

152 alcohol 25:24:1 was performed by shaking gently for 10 minutes and centrifuging at

$15316000 \mathrm{~g}$ for 10 minutes. DNA was precipitated using cold isopropanol for $3 \mathrm{~h}$ at $-20^{\circ} \mathrm{C}$,

154 and a centrifugation step at $16000 \mathrm{rpm}$ for $20 \mathrm{~min}$

155 Quality was estimated using agarose gel and nanodrop analysis. Raw

156 metagenomic data were obtained by paired-end Illumina HiSeq ${ }^{\mathrm{TM}} 2500$ sequencing

157 technology (Baseclear). Original reads were deposited at the National Center for

158 Biotechnology Information (NCBI). 


\section{G. berlandieri metagenome analysis}

161

Illumina paired-end reads were adapter- and quality-trimmed using Trim Galore (17), with a Phred score cutoff of 20. Megahit (18), MetaSpades (19) and IDBA-UD (20) programs where employed to generate three metagenome assemblies. Due to our gene-centric interest, in the search of nitrogen-fixation related genes, high confidence contigs and assembly of a substantial proportion of the dataset was preferred, instead of larger contigs as usual for genome-based approaches (21). Therefore, after quality assessment by QUAST, the more convenient assembly was selected for further analysis using as selection criteria the total length of the assembly and number of contigs $(>=$ $1000 \mathrm{bp})$.

Taxonomic classification and functional annotation of the trimmed reads were carried out by Kaiju (17), using the non-redundant protein database: bacteria, archaea, viruses, fungi and microbial eukaryotes database (NCBI BLAST nr+euk) and visualized with Krona (22), and HUMAnN2 -The HMP Unified Metabolic Analysis Network- (23), respectively. Gene prediction and annotation was carried out by PROKKA (24). In addition, Blast (Buhler et al., 2007) was used to compare taxonomic predictions with reference genomes, in order to refine the results and avoid errors due to incorrectly annotated sequences. The search of nitrogen-fixing related genes was carried out by Blast (11)with nif genes from NCBI, using tblastn with an E-value 1e50 , to obtain a small number of hits with high quality. In addition, blastn was used to identify virulence and related genes in the metagenome with the same e-value, and only those with an identity $\geq 95 \%$ over an alignment length were retained, reference nucleotide sequences were downloaded from EMBL (25). 
185

186

187

188

189

190

191

192

193

194

195

196

197

198

199

200

201

202

203

204

205

\section{Exo-metabolites identification}

K. variicola type strain F2R9 and the bacterial isolate KGB_ 5 obtained from $G$. berlandieri were grown on liquid minimal medium and nitrogen free minimal medium, under microaerobic conditions. Culture supernatants were centrifuged and the extracellular medium lyophilized. These freeze-dryed samples were dissolved in 600 $\mu \mathrm{l}$ of $\mathrm{D}_{2} \mathrm{O}$ sodium phosphate buffer $0.123 \mathrm{M}$ at $\mathrm{pH} 7.4$ with trimethylsilylpropionic acid (TSP) 1mM as internal standard. An Avance III HD 700700 MHz NMR spectrometer equipped with a 5-mm z-axis gradient TCI cryogenic probe (Bruker, Fällanden, Switzerland) was used at $298 \mathrm{~K}$ and ${ }^{1} \mathrm{H}$ frequency of $699.95 \mathrm{MHz} .{ }^{1} \mathrm{H}$ NMR Spectra acquisition were carried out by using the standard NOESY-1D pulse sequence (Bruker program noesyprld), which suppresses water preserving the intensity of most of the lasting signals (26). Water signal was irradiated during relaxation delay (RD): $4.0 \mathrm{~s}$ and mixing time: $10 \mathrm{~ms}, 256$ scans with spectral width: $14 \mathrm{kHz}, 64 \mathrm{k}$ data points, acquisition time: $2.3 \mathrm{~s}$ and exponential line-broadening factor: $0.3 \mathrm{~Hz}$ was applied to the free induction decays (FID) before Fourier transformation. Topspin v 3.5.6 and MestReNova v. 12.0 (MestreLab Research SL.) were used for recording and processing the spectrum (phase and baseline), respectively. TSP chemical shift referenced to 0.000 ppm and Chenomx NMR Suite v. 8.31 (Chenomx Inc.) was used for identification and quantification of the exo-metabolites. 


\section{Results}

\section{Nitrogen-fixing bacteria isolation and acetylene reduction}

\section{assays}

ARA was detected in few fecal samples from herbivorous animals in the Zoofari zoo in Morelos, Mexico. Among them, C. sulcata tortoise stool sample was positive for

211 ethylene production in the ARA assay. Further analysis with an extended sampling of 212 tortoise stools showed that acetylene reduction was obtained in all stools tested and in 213 cultures from the isolates from both G. berlandieri and C. sulcate samples (Table 1, Fig 214 1). In addition, one of the food samples of $G$. berlandieri was positive for ARA assay, 215 using sucrose as carbon source (12.02 $\mathrm{nmol} \mathrm{C}_{2} \mathrm{H}_{4} / \mathrm{h}$ vial).

Table 1. Acetylene reduction activity in G. berlandieri and C. sulcata stools.

\begin{tabular}{|l|l|l|l|}
\hline \multirow{2}{*}{ Sample } & \multicolumn{3}{c|}{ Acethylene reduction activity (nmol $\mathbf{C}_{2} \mathbf{H}_{4} / \mathbf{h}_{\text {vial })}$} \\
\cline { 2 - 4 } & Glucose & Sucrose & D-gluconic acid sodium salt \\
\hline G. berlandieri stool & $167.96 \pm 36.23$ & $222.72 \pm 25.71$ & $57.56 \pm 20.66$ \\
\hline C. sulcata stool & $8.31 \pm 5.83$ & $37.46 \pm 5.26$ & $0.56 \pm 0.19$ \\
\hline K. variicola F2R9 & $4.23 \pm 0.64$ & $30.41 \pm 0.56$ & $7.78 \pm 1.37$ \\
\hline K. variicola VI & $13.77 \pm 4.81$ & $56.38 \pm 7.38$ & $12.82 \pm 1.83$ \\
\hline
\end{tabular}

219 Nitrogen-fixing strains demonstrated the variation in the amount of acetylene reduced

220 according to the available carbon source (Fig 1). In plant nitrogen-fixing symbioses,

221 carbon supplies have been found to limit nitrogen fixation. Previously, it was reported 222 that E. coli mutants affected in gluconate catabolism had a reduced gut colonization 223 (27). Thus we tested if gluconate supported nitrogen fixation in klebsiella and found 224 that it was not the best substrate, seemingly a better supply was sucrose. 
Fig 1. Acetylene reduction activity of bacterial grown in nitrogen-free medium supplemented with different carbon sources.

A total of 27 bacterial isolates were obtained from $G$. berlandieri (KGB) and $C$. sulcata (KCS) stool samples, 24 and 3, respectively. ARA test showed that 25 of them presented detectable nitrogen fixation by GC, and they exhibited identical colony morphology to K. variicola reference strains. According to the ERIC test, six different 235 patterns were observed, and most of them presented the same fingerprints. The patterns 236 of representative strains is shown in Fig 2, fifteen strains from G. berlandieri also 237 presented the same pattern as KGB_1 (including KGB_6 shown in Fig 3), three strains 238 from G. berlandieri exhibited the same fingerprint as KGB_3 (including KGB5 shown 239 in Fig 3) and one more strain from C. sulcata presented KCS1 pattern. Strains KGB2, $240 \mathrm{KCS} 2$ and KGB4 represent each one an unique pattern. In addition, a bacterial isolated 241 from food sample (KFOOD) of $G$. berlandieri also presented acetylene reduction 242 activity, but exhibited a different genomic fingerprint (ERIC pattern) from those in 243 feces. Representative strains of each group were selected for 16s rDNA gene 244 sequencing and acetylene reduction assays. 


\section{Phylogenetic analysis of nitrogen-fixing bacteria using $16 \mathrm{~S}$}

\section{rRNA gene sequences}

The phylogenetic tree obtained indicated that the closest bacteria species to the nitrogen-fixing isolates from $G$. berlandieri and C. sulcata is $K$. variicola for most of the strains, and $K$. michiganesis and $K$. oxytoca for one of the isolates from $C$. sulcata and the strain isolated from the food of G. berlandieri (Fig 3), which was congruent with fingerprints found by BOX-PCR. The $16 \mathrm{~S}$ rRNA gene sequence analysis of the non-nitrogen-fixing isolated strains revealed that they were close to Raoultella ornithinolytica and Klebsiella pneumoniae (not shown).

Fig 3. Phylogenetic tree of $16 \mathrm{~S}$ rRNA gene sequences of representative nitrogenfixing bacterial isolates from $G$. berlandieri $(K G B)$ and $C$. sulcata $(K C S)$ stools, food of $G$. berlandieri (KFOOD) and reference strains.

From a single stool sample, different Klebsiella strains were isolated but remarkably from different tortoise samples common strains were obtained. 
bioRxiv preprint doi: https://doi.org/10.1101/666818; this version posted June 10, 2019. The copyright holder for this preprint (which was

not certified by peer review) is the author/funder, who has granted bioRxiv a license to display the preprint in perpetuity. It is made available under aCC-BY 4.0 International license.

and Amp100, and susceptible to Cb100 and Nal20. In general, most of the nitrogen-

275 fixing isolated strains presented the same antibiotic susceptibility pattern as K. variicola

276 F2R9.

\section{DNA extraction and metagenome sequencing}

Illumina Hiseq2500 paired-end sequencing of a DNA sample obtained from $G$.

berlandieri stool was performed. A total of $84,971,762$ read pairs were generated with

\section{G. berlandieri metagenome analysis}

After trimming, $99.4 \%$ of the original reads were kept for subsequent analysis, corresponding to 84,464,617 cleaned read pairs. The generated MetaSpades, IDBA-UD and MegaHit assembies consisted of 92799, 75327 and 98289 contigs ( $>=1000 \mathrm{bp}$ ), respectively; accounting for 388140063, 271529393 and 393249585 total length $(>=$ $1000 \mathrm{bp}$ ), in each case. Accordingly, Megahit assembly was selected for further

291 identification of nitrogen fixation-related genes in G. berlandieri gut microbiota. Due to, Megahit generated the largest assembly with more contigs of size $>=1000 \mathrm{bp}$.

295 Berlandieri is dominated by bacteria (97\%) with a prevalence of the Firmicutes fylum 296 (53\%), followed by bacteroidetes (10\%) and Proteobacteria (9\%), in which the most 297 abundant class is Gammaproteobacteria (4\%); predominantly shaped by bacteria of the 
order enterobacteriales $(1 \%)$, the family Enterobacteriaceae $(0.9 \%)$ and the genus $(0.07 \%)$ were also detected.

303 (NCBI reference sequence: NR_025635.1) against the metagenome was conducted with blastn obtaining an identity $99.7 \%$, this was the closest species to most of the bacterial isolates in the phylogenetic tree.

metagenome of $\boldsymbol{G}$. berlandieri. A. showing the relative abundance of bacteria, archaea,

314 abundances were carried out using HUMAnN2. A total of 485 pathways were identified

315 in the gut metagenome of $G$. berlandieri, of which 240 were present in klebsiellas (S2

316 Table). Biosynthetic pathways for most of amino acids such as L-arginine, L-histidine,

317 L-lysine, L-aspartate, L-asparagine, cysteine, L-serine, glycine, L-proline, L-threonine,

318 L-methionine, L-tryptophan and L-alanine were found in the metagenome. Among the 319 most abundant pathways for klebsiella are those involved in peptidoglycan maturation, 320 glycolysis, L-1,2-propanediol degradation, myo-,chiro-and scillo-insolitol degradation,

321 L-methionine biosynthesis and fatty acids oxidation. In addition, pathways involved in 322 sucrose degradation; hexitol fermentation to lactate, formate, ethanol and acetate; 
323 amino acids biosynthesis; synthesis of UMP and nitrate reduction; pyruvate

324 fermentation to isobutanol; and others, were found in klebsiella. The biosynthesis of L-

325 alanine was predominantly attributed to klebsiella, followed by Enterobacter,

326 Raoultella and Oxalobacter. Similarly, L-phenylalanine pathway is more abundant in

327 klebsiella, followed by Acinetobacter and Aeromonas.

Metagenome annotation by Prokka predicted a total of 6921 different proteins,

330 excluding the hypotheticals. Some of them are related to catabolism, anabolism, and

331 transport of nutrients and biomolecules, regulation, DNA repair, nitrogen fixation,

332 virulence and antibiotic resistance, among others.

Coding sequences of nitrogen-fixing genes from different species were blast to

335 the metagenome assembly using tblastn. Non-virus, archaea or protist genes related to

336 nitrogen-fixation were found in the microbiome of $G$. berlandieri. According to the

337 tblastn analysis this property may be attributed to genes belonging to bacteria, most of

338 them from the genera Klebsiella. In addition, the twenty genes of the Nif complex in

339 Klebsiella variicola were found in the metagenome assembly by blastn (Table 2).

Table 2. Nif genes of Klebsiella variicola found in G. berlandieri gut

342 metagenome by blastn.

\begin{tabular}{|c|c|c|}
\hline Genes & EMBL ID & Identity \\
\hline nifJ & PXL59115 & $99.15 \%$ \\
\hline nifH & CEL84131 & $99.32 \%$ \\
\hline nifD & CEL84130 & $99.72 \%$ \\
\hline nifK & AHF81417 & $98.85 \%$ \\
\hline nifT & PLP44158 & $99.543 \%$ \\
\hline
\end{tabular}




\begin{tabular}{|c|c|c|}
\hline nifY & CEL84127 & $99.82 \%$ \\
\hline nifE & APW87395 & $98.84 \%$ \\
\hline nifN & PXL56507 & $99.06 \%$ \\
\hline nifX & PLP47623 & $99.58 \%$ \\
\hline nifU & CEL84123 & $99.76 \%$ \\
\hline nifS & PLP47625 & $99.67 \%$ \\
\hline nifV & CEL84121 & $99.74 \%$ \\
\hline nifW & CEL84120 & $99.61 \%$ \\
\hline nifZ & CEL84119 & $98.64 \%$ \\
\hline nifM & PLP48656 & $99.39 \%$ \\
\hline nifF & PLM94490 & $99.62 \%$ \\
\hline nifL & PLP45200 & $99.13 \%$ \\
\hline nifA & REI93249 & $99.11 \%$ \\
\hline nifB & PXL56497 & $99.08 \%$ \\
\hline nifQ & CEL84113 & $98.06 \%$ \\
\hline & & \\
\hline & & \\
\hline
\end{tabular}

A total of 174 genes associated to virulence in Klebsiella were aligned with the

345 metagenome assembly using blastn (29-35). According to it, 38 putative virulence

346 genes were found in the metagenome assembly using as selection criteria an identity up 347 to $95 \%$ throughout all the gene sequence (Table 3), of which 22 correspond to $K$. 348 variicola, 15 belong to $K$. pneumoniae and 1 to $K$. aerogenes. The complete list of 349 studied genes is presented as S3 Table. 


\begin{tabular}{|c|c|c|}
\hline Genes & EMBL ID & Identity \\
\hline \multicolumn{3}{|l|}{ Klebsiella variicola } \\
\hline $\operatorname{luxR}$ & RUP58537 & $100.00 \%$ \\
\hline$m r k F$ & ACI09593 & $100.00 \%$ \\
\hline$m r k B$ & CEP28159 & $99.86 \%$ \\
\hline fimH & ACI06808 & $99.78 \%$ \\
\hline fur & SXF53026 & $99.78 \%$ \\
\hline pcoE & CTQ05476 & $99.78 \%$ \\
\hline$a c r B$ & OYD22191 & $99.67 \%$ \\
\hline$m d t K$ & PLK35013 & $99.64 \%$ \\
\hline$m r k C$ & ACI10676 & $99.44 \%$ \\
\hline ureD & OYD24111 & $99.39 \%$ \\
\hline fepC & OUG52026 & $99.36 \%$ \\
\hline Salmochelin siderophore protein iroE & OWW17284 & $99.25 \%$ \\
\hline phoQ & OWW16474 & $99.18 \%$ \\
\hline$y c f M$ & SXE98705 & $99.07 \%$ \\
\hline$f e o B$ & PLK36930 & $98.66 \%$ \\
\hline phoP & VGP77699 & $98.66 \%$ \\
\hline uge & EFD86038 & $98.31 \%$ \\
\hline ent $B$ & VGQ02355 & $98.24 \%$ \\
\hline pcoC & ROG35707 & $97.90 \%$ \\
\hline$m r K D$ & CEL88440 & $97.89 \%$ \\
\hline traT & RUP54965 & $97.26 \%$ \\
\hline acrA & VGP78838 & $96.04 \%$ \\
\hline \multicolumn{3}{|l|}{ Klebsiella pneumoniae } \\
\hline$p c o E$ & OWA97968 & $100.00 \%$ \\
\hline$p c o A$ & AEV55124 & $99.31 \%$ \\
\hline$l r p-1$ & ALR23660 & $98.99 \%$ \\
\hline$p c o C$ & EWC99993 & $97.90 \%$ \\
\hline entB & AYU88760 & $97.27 \%$ \\
\hline$m r k B$ & AFV70429 & $97.15 \%$ \\
\hline ureA & ALR25992 & $97.03 \%$ \\
\hline iroN & ABR76677 & $97.02 \%$ \\
\hline fur & AAB51077 & $97.02 \%$ \\
\hline$f e o B$ & PAX11144 & $96.25 \%$ \\
\hline$m r k A$ & AFV70428 & $95.94 \%$ \\
\hline $\mathrm{fimH}$ & ACL13776 & $95.82 \%$ \\
\hline$w a b G$ & AAX20104 & $95.66 \%$ \\
\hline acrA & CAC41008 & $95.16 \%$ \\
\hline$y c f M$ & BBE67743 & $93.98 \%$ \\
\hline \multicolumn{3}{|l|}{ Klebsiella aerogenes } \\
\hline ureD & AAA25148 & $96.06 \%$ \\
\hline
\end{tabular}




\section{Exo-metabolites identification}

A total of 31 molecules were identified in the supernatant cultures of the $G$.

358 berlandieri nitrogen-fixing strain KGB_5, K. variicola F2R9 growth separately under

359 a microaerobic environment, in the presence and absent of a nitrogen source (S4 Table).

360 Here, we found alanine as an exo-metabolite of KGB_5 in both nitrogen-containing and

361 nitrogen-free medium by $1 \mathrm{H}-\mathrm{NMR}$, as well as in the extracellular culture medium of $K$.

362 variicola F2R9 in nitrogen-containing minimal medium. A trace amount of alanine was

363 detected in the nitrogen-containing minimal medium, with a concentration more than

36430 times lower than the found in bacterial cultures grown in this medium.

Under nitrogen-free conditions, a similar metabolic footprinting was observed

368 both strains were: 2-hydroxyisovalerate, 2-oxoglutarate, 4-hydroxyphenylacetate, 369 betaine, fumarate, phenylacetate, pyruvate, 3-phenyllactate. However, alanine was 370 solely detected in the extracellular culture of KGB_5; and malonate in K. variicola $371 \quad$ F2R9 (Fig 5).

Fig 5. ${ }^{1} \mathrm{H}-\mathrm{NMR}$ spectra of culture supernatants of A) bacterial isolated

376 5: fumarate; 6: sucrose; 8: glucose; 9: 1,3-dihydroxyacetone; 10: lactate; 12: glycolate;

377 13: betaine; 15: methanol; 16: malonate; 17: dimethyl sulfone; 19: 2-oxoglutarate;

378 20: N,N-dimethylglycine; 21: dimethylamine; 22: succinate; 23: pyruvate; 24: acetone;

379 25: acetate; 26: alanine; 28: 3-hydroxybutyrate; 29: ethanol; 30: 2-hydroxyisovalerate and 31: 2-hydroxyvalerate. 
were released by both the isolated KGB_5 and $K$. variicola F2R9: 1,3-diaminopropane, and pyruvate. The molecules that were only detected from KGB_5 under this condition were: 3-hydroxybutyrate, fumarate, 2-hydroxyisobutyrate, glycerate and maltose. On the other hand, phenylacetate and 2-hydroxyvalerate were detected in the extracellular medium of $K$. variicola F2R9 grown in nitrogen-containing minimal medium (Fig 6).

Fig 6. ${ }^{1} \mathrm{H}-\mathrm{NMR}$ spectra of culture supernatants of A) bacterial isolated

KGB_5, B) K. variicola F2R9 grown in C) nitrogen-containing minimal medium.

\section{Discussion}

403 humans with different diets, however the microbiota from tortoises had not been reported.

404 Insects that feed on low nitrogen and high carbon diets profit from nitrogen-fixing symbioses

405 with distinct gut bacteria. Termites are the best known examples, but wood eating beetles also 
406 harbor diazotrophs. Nitrogen-fixing symbioses had not been described in reptiles and may help

407 to compensate nitrogen deficiencies in their diet.

\section{Nitrogen-fixing bacteria in tortoise feces}

Culture-dependent and independent approaches revealed that the enterobacteria $K$.

variicola is found in G. berlandieri. From C. sulcata, K. variicola was isolated from feces in

culture media as well. Here fecal bacterial isolation was in microaerobic nitrogen-free medium to enrich for nitrogen-fixing bacteria. This allowed the isolation of nitrogen-fixing but few nonnitrogen-fixing bacteria were obtained as well that were identified as Rhanella. corresponded to K.variicola.

K. variicola is a nitrogen-fixing species closely related to K. pneumoniae (36,

37) which rarely fixes nitrogen. The former has been isolated from plants such as maize,

sugarcane and banana, as a plant-growth-promoting root endophyte (inside plant patients, in special from babies. Klebsiellas are common colonizers of human intestine.

that $K$. variicola is pathogenic in tortoises as they were healthy and we suppose that they are mutualists as occurs in plants. Here we surmise that nitrogen fixation indeed occurs in tortoise guts on the basis of the ethylene reduction activity that we detected 
433 genes than clinical isolates (36), here we found that klebsiellas from feces were 434 susceptible to $\mathrm{Cb} 100$ and Nal20, though they were resistant to ampicillin. All $K$. 435 variicola strains are resistant to ampicillin and the gene responsible is chromosomally 436 encoded indicating that is not easily lost or transferred. Previously we proposed that 437 there exists a different mode of transmission for $K$. variicola and $K$. pneumoniae with 438 K. variicola acquired from the environment and $K$. pneumoniae transmitted from 439 human to human $(36,40)$. If tortoise klebsiellas are transmitted from animal to animal, 440 then better gut adapted strains (see below) may be selected avoiding infection with 441 pathogenic bacteria. Indeed we found that $K$. variicola from tortoises had a low 442 proportion of virulence determinants.

\section{Origin of tortoise klebsiellas}

Bacteria may be obtained from feces ingestion as coprophagy is a common 446 practice among some tortoises or they may derive from their vegetable diet as $K$. 447 variicola strains are commonly found in plants. To address this we isolated bacteria 448 associated from the vegetables used for feeding the Texas tortoises or the pellets used 449 to feed the African tortoise. All plants contain endophytes and there is a large diversity 450 of endophytes which may be recovered from plants after a surface disinfection 451 procedure that eliminates bacteria that are not within tissues. We suppose that by being 452 endophytes, klebsiellas are protected in the inside of vegetable tissues and may survive 453 the digestion process. Since herbivorous animals exhibit the largest diversity of gut 454 bacteria in comparison to ommivorous or carnivorous animals (41), it seems that 455 endophytes may become constituents of gut microbiota, at least transiently. 456 Alternatively, common klebsiella symbionts among tortoises would indicate that they 
457 are vertically transmitted and well adapted to hosts. We found that diet and gut nitrogen-

458 fixing bacteria were not the same, indicating that there is not a direct transfer of

459 nitrogen-fixing bacteria from the ingested food. If klebsiellas are not obtained from the

460 diet, then gut klebsiellas may be obtained by coprophagy in tortoises and perhaps early

461 in life. It is remarkable that fecal klebsiella from distinct individual tortoises are very

462 similar from the REP-BOX study performed (that allows strain distinction), the limited

463 diversity encountered would favor the tortoise-tortoise transmission hypothesis.

464 It is worth considering that there could be some negative aspects of nitrogen

465 fixation by Klebsiella in guts. Antibodies against nitrogenase have been detected in

466 humans with ankylosing spondylitis and Klebsiella infections have been considered as

467 candidates for the onset of such disease (42). We wonder if tortoises would have as well

468 an immune response towards klebsiella nitrogenase and what the effects in their heath 469 could be.

471 found in the metagenome analysis of $G$. berlandieri.

\section{Exo-metabolite analysis}

473 Diazotrophs such as Klebsiella that are among the free- living nitrogen-fixing bacteria normally do not excrete ammonium produced by nitrogen fixation and use it for their own bacterial growth. To further explore this we analyzed the metabolites excreted by

476 a K. variicola isolate in vitro under microaerobic conditions in nitrogen free medium

477 in comparison to medium with added nitrogen. By 1H-NMR exo-metabolite analysis, 478 alanine was detected in the supernatant of $K$. variicola KGB_5 grown in nitrogen free 479 medium and microaerobic conditions while the plant $K$. variicola F2R9 strain did not 480 excrete alanine under those conditions. Interestingly some Rhizobium strains may 481 provide alanine to plants in symbiosis $(44,45)$. It would be worth exploring if alanine 
excretion is a common adaptative characteristic of tortoise klebsiellas and not particular

483 to the tested strain. In addition, a number of exo-metabolites were produced by both

484 KGB_5 and K. variicola F2R9 under nitrogen-containing minimal medium and 485 nitrogen free conditions.

486

\section{Acknowledgements}

To Michael Dunn for reading the manuscript.

\section{Funding Sources}

This research was supported by Universidad Autónoma de México [Grant:

491 Programa de Becas Posdoctorales en la UNAM 2016 to D. M-G], CONACyT [Grant:

492 253116] and PAPIIT [Grant: IN207718]. This study made use of UNAM's NMR lab:

493 LURMN at IQ-UNAM, which is funded by CONACYT Mexico (Project: 0224747), 494 and UNAM.

495

496 References

497 1. Halbleib CM, Ludden PW. Regulation of biological nitrogen fixation. J Nutr. $498 \quad 2000 ; 130(5): 1081-4$.

499 2. Benemann JR, Valentine R. The pathways of nitrogen fixation. Advances in 500 Microbial Physiology: Elsevier; 1972. p. 59-104.

501 3. Kundu DK, Ladha JK. Enhancing Soil Nitrogen Use and Biological Nitrogen

502 Fixation in Wetland Rice. Experimental Agriculture. 1995;31(3):261-78. 
503 4. Morales-Jiménez J, de León AV-P, García-Domínguez A, Martínez-Romero E,

504 Zúñiga G, Hernández-Rodríguez C. Nitrogen-fixing and uricolytic bacteria associated

505 with the gut of Dendroctonus rhizophagus and Dendroctonus valens (Curculionidae:

506 Scolytinae). Microbial ecology. 2013;66(1):200-10.

507 5. Pinto-Tomás AA, Anderson MA, Suen G, Stevenson DM, Chu FS, Cleland

508 WW, et al. Symbiotic nitrogen fixation in the fungus gardens of leaf-cutter ants.

509 Science. 2009;326(5956):1120-3.

510 6. Vera-Ponce de León A, Ormeño-Orrillo E, Ramírez-Puebla ST, Rosenblueth

511 M, Degli Esposti M, Martínez-Romero J, et al. Candidatus Dactylopiibacterium 512 carminicum, a nitrogen-fixing symbiont of Dactylopius cochineal insects (Hemiptera:

513 Coccoidea: Dactylopiidae). Genome Biol Evol. 2017;9(9):2237-50.

$5147 . \quad$ Judd FW, Rose FL. Population structure, density and movements of the Texas 515 tortoise Gopherus berlandieri. Southwest Nat. 1983:387-98.

516 8. Rose FL, Judd FW. Activity and home range size of the Texas tortoise, 517 Gopherus berlandieri, in south Texas. Herpetologica. 1975:448-56.

518 9. Petrozzi F, Hema EM, Luiselli L, Guenda W. A survey of the potential 519 distribution of the threatened tortoise Centrochelys sulcata populations in Burkina Faso 520 (West Africa). Tropical Ecology. 2016;57(4):709-16.

521 10. Abdelrhman KF, Bacci G, Mancusi C, Mengoni A, Serena F, Ugolini A. A first 522 insight into the gut microbiota of the sea turtle Caretta caretta. Front Microbiol. $5232016 ; 7: 1060$.

524 11. Johnson M, Zaretskaya I, Raytselis Y, Merezhuk Y, McGinnis S, Madden TL. 525 NCBI BLAST: a better web interface. Nucleic Acids Res. 2008;36:W5-W9.

526 12. Katoh K, Rozewicki J, Yamada KD. MAFFT online service: multiple sequence 527 alignment, interactive sequence choice and visualization. Brief Bioinform. 2017. 
528 13. Santorum JM, Darriba D, Taboada GL, Posada D. Jmodeltest. org: selection of 529 nucleotide substitution models on the cloud. Bioinformatics. 2014;30(9):1310-1.

530 14. Guindon S, Dufayard J-F, Lefort V, Anisimova M, Hordijk W, Gascuel O. New 531 algorithms and methods to estimate maximum-likelihood phylogenies: assessing the 532 performance of PhyML 3.0. Syst Biol. 2010;59(3):307-21.

533 15. Kumar S, Stecher G, Li M, Knyaz C, Tamura K. MEGA X: Molecular 534 Evolutionary Genetics Analysis across Computing Platforms. Mol Biol Evol. $535 \quad 2018 ; 35(6): 1547-9$.

536 16. Zhang B-W, Li M, Ma L-C, Wei F-WJBg. A widely applicable protocol for 537 DNA isolation from fecal samples. Biochem Genet. 2006;44(11-12):494.

538 17. Krueger F. Trim galore. A wrapper tool around Cutadapt and FastQC to 539 consistently apply quality and adapter trimming to FastQ files. 2015.

540 18. Palama TL, Canard I, Rautureau GJ, Mirande C, Chatellier S, Elena-Herrmann 541 B. Identification of bacterial species by untargeted NMR spectroscopy of the exo542 metabolome. Analyst. 2016;141(15):4558-61.

543 19. Nurk S, Meleshko D, Korobeynikov A, Pevzner PA. metaSPAdes: a new 544 versatile metagenomic assembler. Genome Res. 2017:gr. 213959.116.

545 20. Peng Y, Leung HC, Yiu S-M, Chin FY. IDBA-UD: a de novo assembler for 546 single-cell and metagenomic sequencing data with highly uneven depth. 547 Bioinformatics. 2012;28(11):1420-8.

548 21. Van der Walt AJ, Van Goethem MW, Ramond J-B, Makhalanyane TP, Reva O, 549 Cowan DA. Assembling metagenomes, one community at a time. BMC genomics. $550 \quad 2017 ; 18(1): 521$.

551 22. Ondov BD, Bergman NH, Phillippy AM. Interactive metagenomic visualization 552 in a Web browser. BMC Bioinformatics. 2011;12(1):385. 
553 23. Franzosa EA, McIver LJ, Rahnavard G, Thompson LR, Schirmer M, Weingart

$554 \mathrm{G}$, et al. Species-level functional profiling of metagenomes and metatranscriptomes.

555 Nat Methods. 2018;15(11):962.

556 24. Seemann T. Prokka: rapid prokaryotic genome annotation. Bioinformatics.

$557 \quad 2014 ; 30(14): 2068-9$.

558 25. Stoesser G, Baker W, van den Broek A, Camon E, Garcia-Pastor M, Kanz C, et

559 al. The EMBL nucleotide sequence database. Nucleic Acids Res. 2002;30(1):21-6.

560 26. Le Guennec A, Tayyari F, Edison AS. Alternatives to nuclear overhauser

561 enhancement spectroscopy presat and carr-purcell-meiboom-gill presat for NMR-

562 based metabolomics. Anal Chem. 2017;89(17):8582-8.

563 27. Chang D-E, Smalley DJ, Tucker DL, Leatham MP, Norris WE, Stevenson SJ, 564 et al. Carbon nutrition of Escherichia coli in the mouse intestine. Proc Natl Acad Sci U 565 S A. 2004;101(19):7427-32.

566 28. Menzel P, Ng KL, Krogh AJB. Kaiju: Fast and sensitive taxonomic 567 classification for metagenomics. Biorxiv. 2015:031229.

568 29. Turton JF, Payne Z, Coward A, Hopkins KL, Turton JA, Doumith M, et al. 569 Virulence genes in isolates of Klebsiella pneumoniae from the UK during 2016, 570 including among carbapenemase gene-positive hypervirulent K1-ST23 and 'non571 hypervirulent'types ST147, ST15 and ST383. J Med Microbiol. 2017;67(1):118-28.

572 30. Jian-li W, Yuan-yuan S, Shou-yu G, Fei-fei D, Jia-yu Y, Xue-hua W, et al. 573 Serotype and virulence genes of Klebsiella pneumoniae isolated from mink and its 574 pathogenesis in mice and mink. Sci Rep. 2017;7(1):17291.

575 31. Lopes ACS, Rodrigues JF, Cabral AB, da Silva ME, Leal NC, da Silveira VM, 576 et al. Occurrence and analysis of irp2 virulence gene in isolates of Klebsiella 
pneumoniae and Enterobacter spp. from microbiota and hospital and communityacquired infections. Microb Pathog. 2016;96:15-9.

579 32. Cortés G, de Astorza B, Benedí VJ, Albertí S. Role of the htrA gene in 580 Klebsiella pneumoniae virulence. Infect Immun. 2002;70(9):4772-6.

581 33. Wasfi R, Elkhatib WF, Ashour HM. Molecular typing and virulence analysis of 582 multidrug resistant Klebsiella pneumoniae clinical isolates recovered from Egyptian 583 hospitals. Sci Rep. 2016;6:38929.

584 34. Nahar N, Rashid RB. Phylogenetic Analysis of Antibiotic Resistance Genes and 585 Virulence Genes of Klebsiella species in silico. Dhaka Univ J Pharm Sci. $586 \quad 2017 ; 16(1): 119-27$.

587 35. Andrade BGN, de Veiga Ramos N, Marin MFA, Fonseca EL, Vicente ACP. 588 The genome of a clinical Klebsiella variicola strain reveals virulence-associated traits 589 and a p19-like plasmid. FEMS Microbiol Lett; 2014. p. 13-6.

590 36. Rosenblueth M, Martínez L, Silva J, Martínez-Romero E. Klebsiella variicola, 591 A Novel Species with Clinical and Plant-Associated Isolates. Syst Appl Microbiol. $592 \quad 2004 ; 27(1): 27-35$.

593 37. Martínez-Romero E, Rodríguez-Medina N, Beltrán-Rojel M, Toribio-Jiménez 594 J, Garza-Ramos U. Klebsiella variicola and Klebsiella quasipneumoniae with capacity 595 to adapt to clinical and plant settings. Sal Pub Mex. 2018;60:29-40.

596 38. Rosales-Bravo H, Morales-Torres H, Vázquez-Martínez J, Molina-Torres J, 597 Olalde-Portugal V, Partida-Martínez L. Novel consortium of Klebsiella variicola and 598 Lactobacillus species enhances the functional potential of fermented dairy products by 599 increasing the availability of branched-chain amino acids and the amount of distinctive 600 volatiles. J Appl Microbiol. 2017;123(5):1237-50. 
601 39. Fouts DE, Tyler HL, DeBoy RT, Daugherty S, Ren Q, Badger JH, et al.

602 Complete genome sequence of the N2-fixing broad host range endophyte Klebsiella

603 pneumoniae 342 and virulence predictions verified in mice. PLoS Genet.

$604 \quad 2008 ; 4(7): \mathrm{e} 1000141$.

605 40. Rosenblueth M, Martínez-Romero E. Bacterial endophytes and their 606 interactions with hosts. Mol Plant Microbe Interact. 2006;19(8):827-37.

607 41. O' Donnell MM, Harris HMB, Ross RP, O'Toole PW. Core fecal microbiota of

608 domesticated herbivorous ruminant, hindgut fermenters, and monogastric animals.

609 Microbiologyopen. 2017;6(5):e00509.

610 42. Husby G, Tsuchiya N, Schwimmbeck PL, Keat A, Pahle JA, Oldstone MB, et

611 al. Cross-reactive epitope with Klebsiella pneumoniae nitrogenase in articular tissue of

612 HLA-B27+ patients with ankylosing spondylitis. Arthritis Rheum. 1989;32(4):437-45.

613 43. Costello EK, Gordon JI, Secor SM, Knight R. Postprandial remodeling of the 614 gut microbiota in Burmese pythons. ISME J. 2010;4(11):1375.

615 44. Waters JK, Hughes BL, Purcell LC, Gerhardt KO, Mawhinney TP, Emerich 616 DW. Alanine, not ammonia, is excreted from N2-fixing soybean nodule bacteroids. 617 Proc Natl Acad Sci U S A. 1998;95(20):12038-42.

618 45. Lodwig EM, Hosie AH, Bourdès A, Findlay K, Allaway D, Karunakaran R, et 619 al. Amino-acid cycling drives nitrogen fixation in the legume-Rhizobium symbiosis. $620 \quad$ Nature. 2003;422(6933):722. 
bioRxiv preprint doi: https://doi.org/10.1101/666818; this version posted June 10, 2019. The copyright holder for this preprint (which was

not certified by peer review) is the author/funder, who has granted bioRxiv a license to display the preprint in perpetuity. It is made available under aCC-BY 4.0 International license.

S2 Table. HUMAnN2 functional analysis results.

627 study.

S4 Table. Chemical shifts of the compounds identified by 1H NMR (700

629 MHz, 298K, TSP=1mM, D2O sodium phosphate buffer $0.123 \mathrm{M}$ at $\mathbf{p H}$ 7.4). TSP

630 was used as internal standard and referenced to chemical shift $0.000 \mathrm{ppm}$.

631 


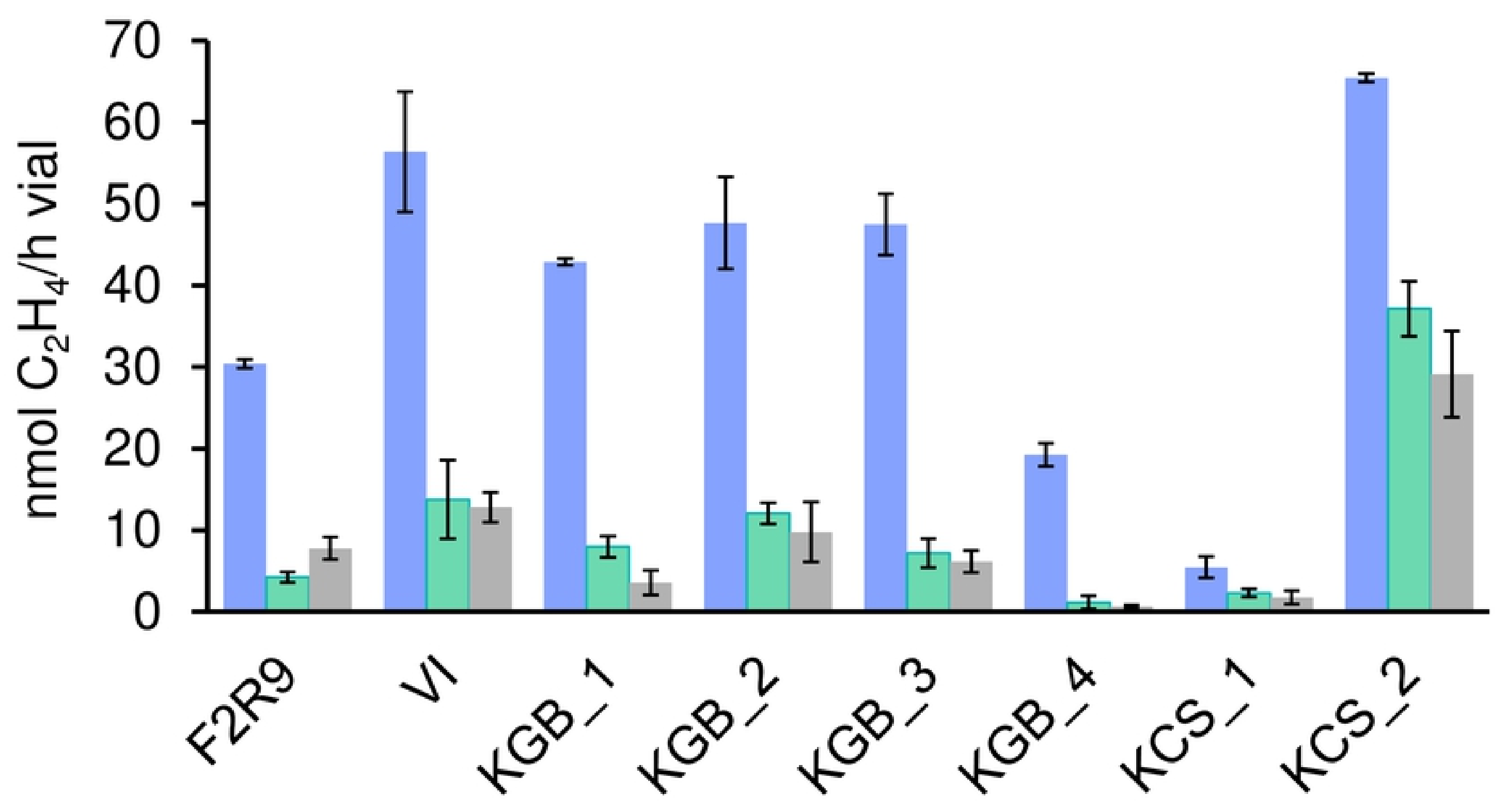

$\square$ Sucrose $\square$ Glucose $\square$ D-gluconic acid sodium salt 


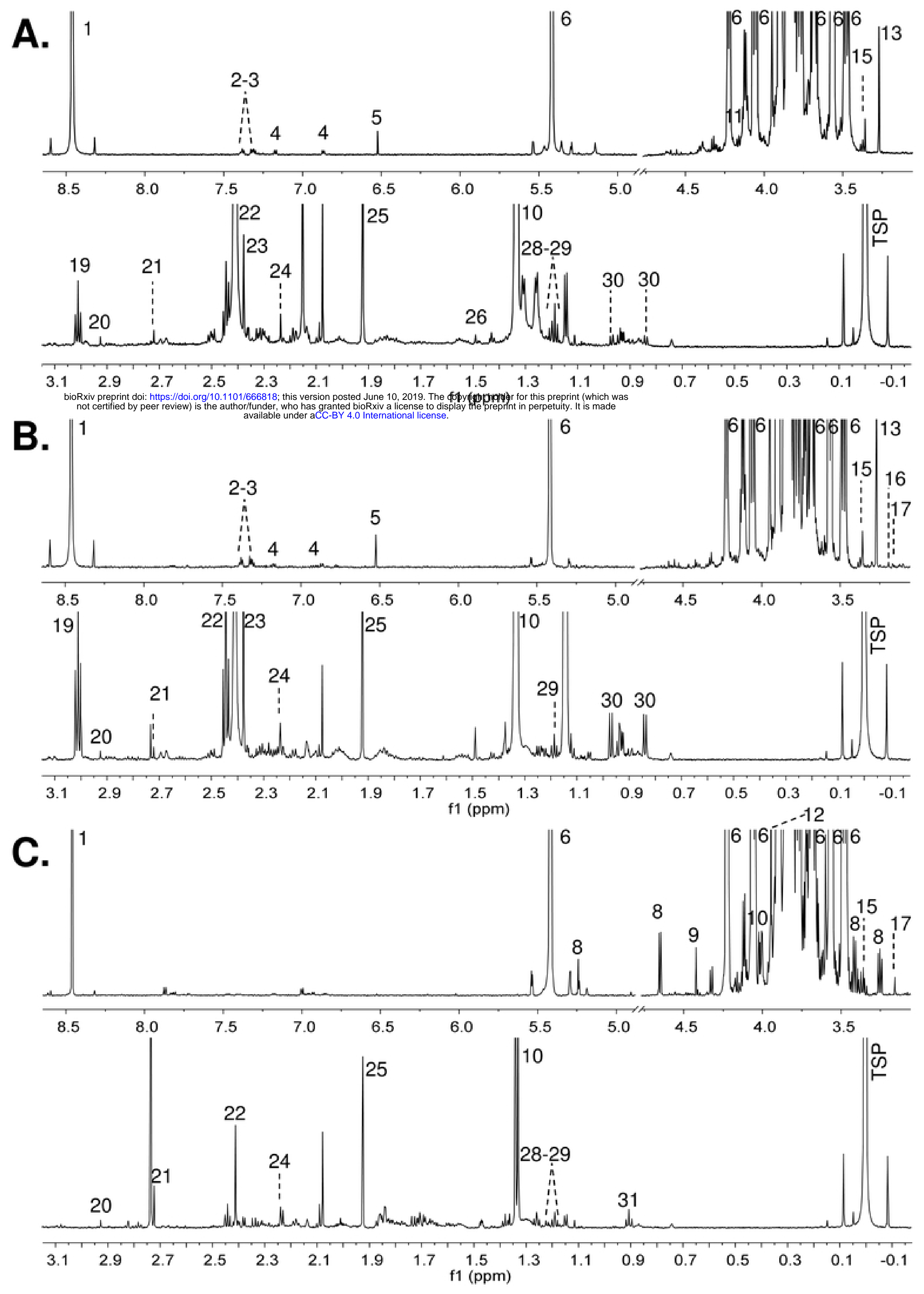




\section{$\begin{array}{lllllllll}M & 1 & 2 & 3 & 4 & 5 & 6 & 7 & 8\end{array}$}

1,200

2,000

1,650

1,000 850

650 500 400 300 200 100 (bp) 
A.

蛋

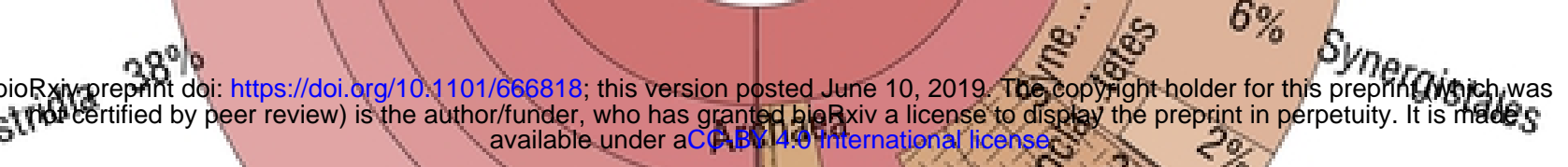
Clost
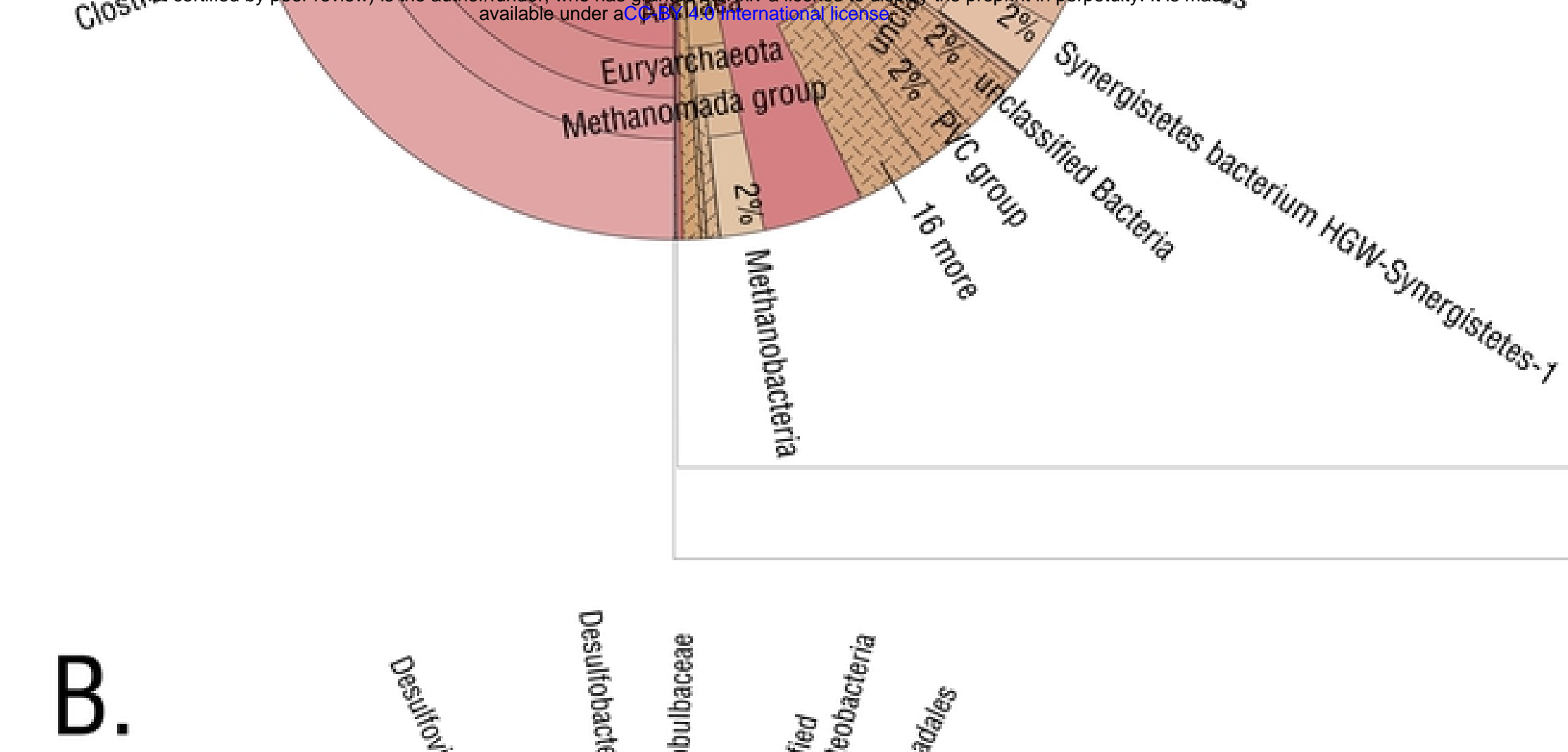

unclassified

Gammaproteobacteria $2 \%$
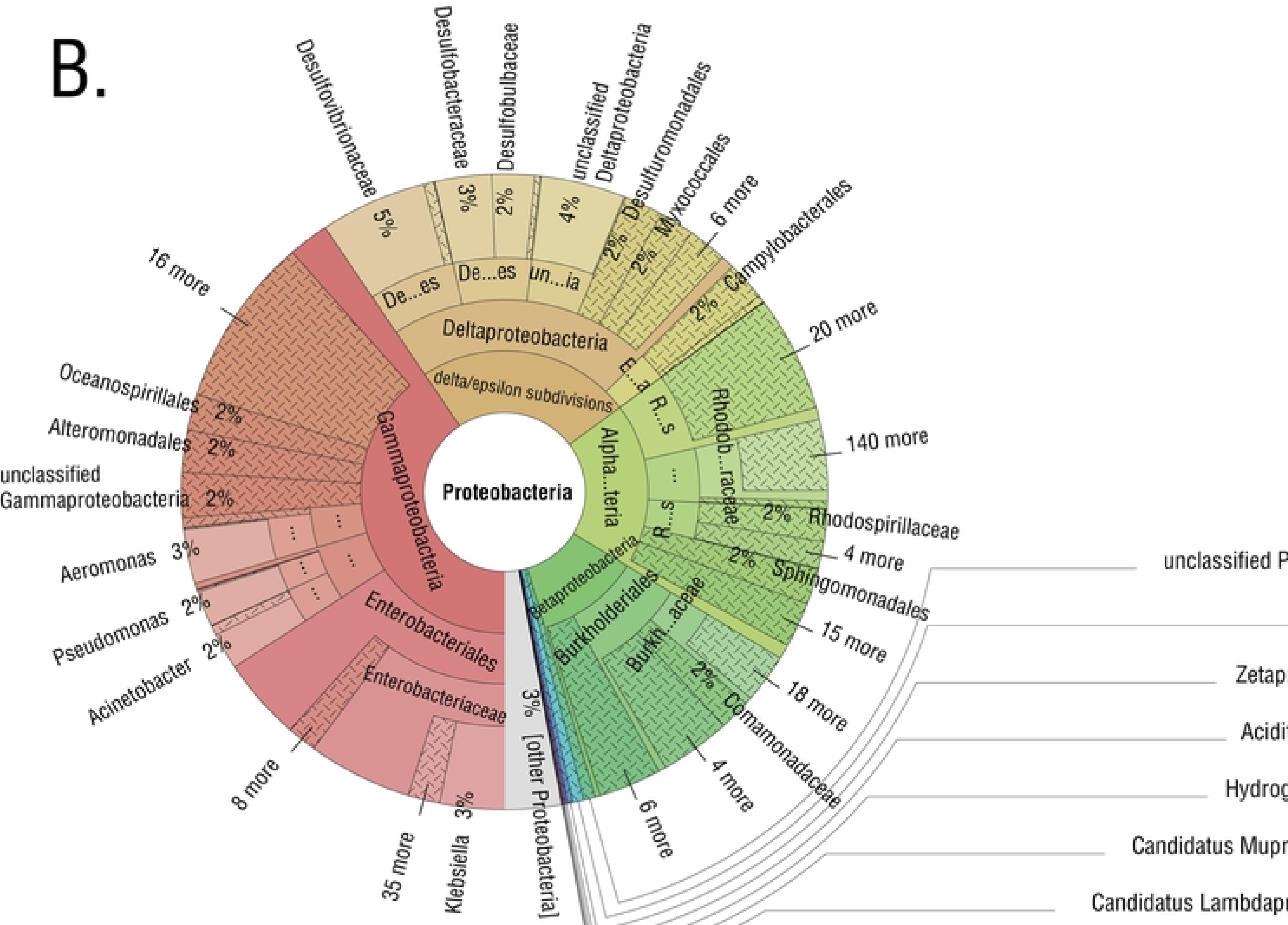


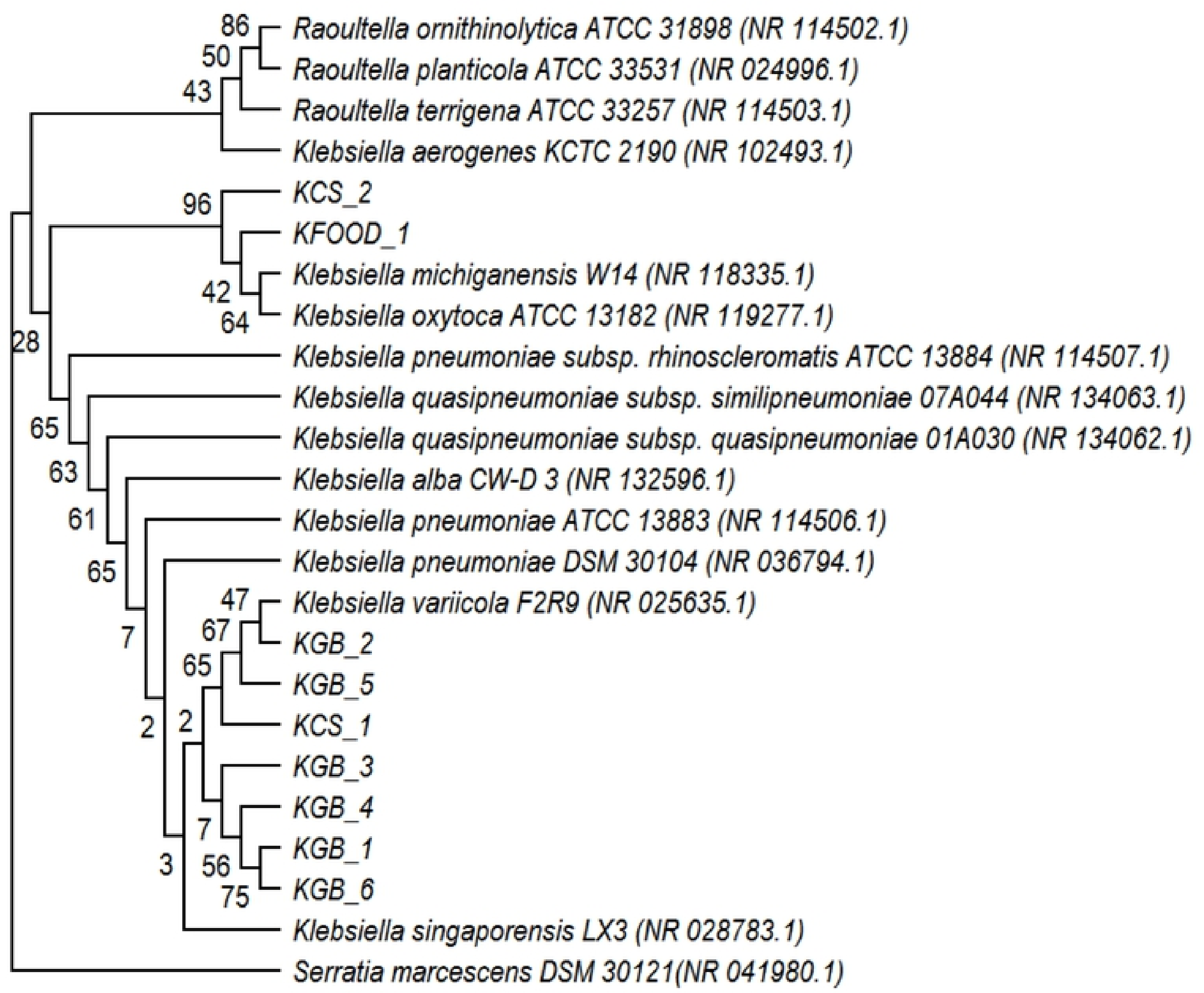

